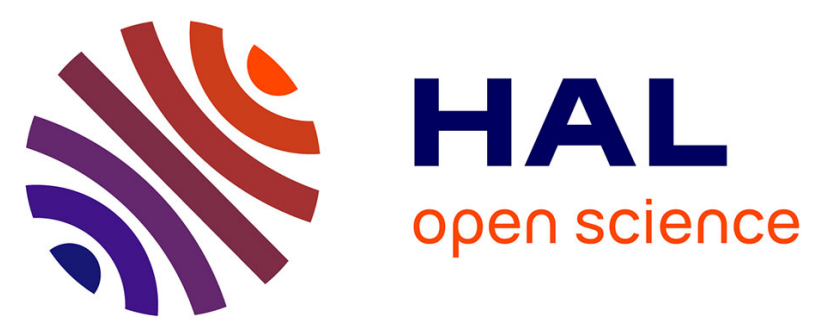

\title{
Clustering and switching processes in semantic verbal fluency in the course of Alzheimer's disease subjects: Results from the PAQUID longitudinal study.
}

Nadine Raoux, Hélène Amieva, Mélanie Le Goff, Sophie Auriacombe, Luc

Letenneur, Jean-François Dartigues

\section{To cite this version:}

Nadine Raoux, Hélène Amieva, Mélanie Le Goff, Sophie Auriacombe, Luc Letenneur, et al.. Clustering and switching processes in semantic verbal fluency in the course of Alzheimer's disease subjects: Results from the PAQUID longitudinal study.: Verbal fluency in pre-clinical AD. Cortex, 2008, 44 (9), pp.1188-96. 10.1016/j.cortex.2007.08.019 . inserm-00180079

\section{HAL Id: inserm-00180079 https://www.hal.inserm.fr/inserm-00180079}

Submitted on 3 Sep 2008

HAL is a multi-disciplinary open access archive for the deposit and dissemination of scientific research documents, whether they are published or not. The documents may come from teaching and research institutions in France or abroad, or from public or private research centers.
L'archive ouverte pluridisciplinaire HAL, est destinée au dépôt et à la diffusion de documents scientifiques de niveau recherche, publiés ou non, émanant des établissements d'enseignement et de recherche français ou étrangers, des laboratoires publics ou privés. 
CLUSTERING AND SWITCHING PROCESSES IN SEMANTIC VERBAL

FLUENCY IN THE COURSE OF ALZHEIMER'S DISEASE SUBJECTS: RESULTS FROM THE PAQUID LONGITUDINAL STUDY

Nadine Raoux ${ }^{a *}$, Hélène Amieva ${ }^{a}$, Mélanie Le Goff ${ }^{a}$, Sophie Auriacombe ${ }^{b}$, Laure Carcaillon $^{\text {a }}$, Luc Letenneur ${ }^{\text {a }}$, Jean-François Dartigues ${ }^{\text {ab }}$.

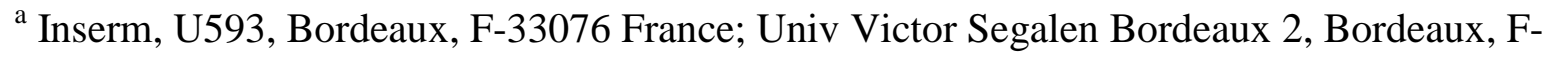
33076 France;

${ }^{\mathrm{b}}$ University Hospital of Bordeaux, France

*Corresponding author: Nadine Raoux. INSERM U593. University Bordeaux 2. 146 Rue Léo Saignat, 33076 Bordeaux Cedex - France. Tel. + 33557574 538; Fax + 33557571486.

E-mail address: Nadine.Raoux@isped.u-bordeaux2.fr

Running title: verbal fluency in pre-clinical AD 


\section{Abstract}

Reduced semantic fluency performances have been reported in the preclinical phase of Alzheimer's disease (AD). To investigate the cognitive processes underlying this early deficit, this study analyzed the verbal production of pre-demented subjects for the animals category with the qualitative parameters related to clustering (i.e. the ability to generate words belonging to semantic subcategories of animals) and switching (i.e. the ability to shift from one subcategory to another) proposed by Troyer.

This qualitative analysis was applied to the PAQUID cohort, a 17-year longitudinal population-based study. The performances on the animal verbal fluency task of 51 incident cases of possible and probable $\mathrm{AD}$ were analyzed at the onset of dementia, 2 years and 5 years before dementia onset. Each case was matched for age, sex and education to two control subjects leading to a sample of 153 subjects. The mean cluster size and the raw number of switches were compared in the two samples. The results revealed a significantly lower switching index in the future AD subjects than in the elderly controls including 5 years before dementia incidence. A significant decline in this parameter was evidenced all along the prodromal phase until the clinical diagnosis of dementia. In contrast, the mean cluster size could not discriminate the two groups. Therefore the results support the hypothesis that impaired shifting abilities -rather than semantic memory storage degradation- could explain the early decline in semantic fluency performance occurring in the predementia phase of AD. 


\section{Introduction}

Verbal fluency tests are frequently used in both clinical examinations and research studies on neurological disorders, including focal lesions, neurodegenerative disorders or psychiatric disorders. Semantic fluency tasks, such as category fluency (e.g. animals or fruits) require the subject to generate exemplars of a given semantic category within a limited time or items which can be bought in a supermarket for the "supermarket task" whereas letter fluency tasks require generation of words beginning by a given letter (e.g. FAS). Alzheimer's disease (AD), often considered as the prototype of cortical dementia, impairs both letter and category fluency tasks but semantic fluency would be altered to a greater extent, as suggested by the meta-analysis by Henry et al. (2004). The use of semantic fluency proves to be of particular interest not only in the dementia phase but also in the preclinical phase of $\mathrm{AD}$ as suggested by a recent prospective population-based study (Amieva et al., 2005). Indeed, the PAQUID study, a French longitudinal population-based study investigated the prodromal period of dementia of the Alzheimer type over a 9-year follow-up. Neuropsychological measures including the Isaacs Set Test (IST) (Isaacs and Kennie, 1973) consisting in evaluating verbal fluency in four semantic categories (animals, fruits, colors and cities) were assessed in 215 future $\mathrm{AD}$ patients and 1050 normal subjects. The results have shown that compared to elderly controls, the IST performance in the patients was already lower 9 years before the clinical diagnosis of $\mathrm{AD}$ and declined regularly all along the prodromal phase until the incidence of dementia syndrome.

In semantic verbal fluency tasks, multiple cognitive processes are at work. First of all, semantic fluency requires the integrity of semantic memory including both undamaged memory storage and preserved access to these semantic stores. When several items within a subcategory of the semantic network are activated, mental flexibility is also important to 
allow the subject to disengage this particular subcategory and reengage a different one in order to shift between semantic subcategories and generate efficient strategies (Troyer et al., 1997). Besides semantic memory and shifting abilities, the ability to efficiently generate as many words as possible within a limited spell of time also involves various executive processes. In particular updating and inhibitory processes of working memory are involved to avoid the recurrence of a response previously produced or to suppress intrusions automatically activated (Auriacombe et al., 2006). Finally, a general speed of processing component is involved due to the time constraints of the task (Abwender et al., 2001; Mayr, 2002).

Therefore the multidetermined nature of semantic fluency makes it difficult to identify which cognitive process is responsible for the early decline in IST performance in AD. A qualitative analysis of subjects' production may be helpful to identify the underlying cognitive dysfunction. A first analysis from the longitudinal Paquid study considered the occurrence of repetitions and intrusions as potential indicators of cognitive dysfunction (Auriacombe et al., 2006). Five years before the clinical diagnosis of AD, the IST score in the predemented subjects was lower than that of control subjects. However when considering the rate of repetitions and intrusions, the results showed that repetitions were not more frequent in the subjects who would develop dementia 5 years later than in normal controls. Concerning intrusions, the rates remained very low and were not different between the predemented and the control groups. The authors concluded that deficits in inhibitory mechanisms regulating working memory could not be considered as the main factor responsible for the early verbal fluency decline, leaving room for further hypotheses such as disorganized retrieval from semantic memory or degraded semantic storage.

Indeed, previous numerous studies have examined the degree to which the words generated during a verbal fluency task are associated or gathered in clusters (Raskin et al., 
1992; Robert et al., 1996). The two-component model of fluency performance proposed by Troyer et al. (1997) has gained considerable popularity in the last decade. According to this model, the optimal performance on fluency tasks relies on two main processes: 1- clustering, a semantic process underlying the production of words within semantic or phonemic subcategories; 2- switching, an executive process underlying the strategic search, cognitive flexibility and shifting between subcategories. According to these authors, clustering would be supported by relatively automatic cognitive processes, while switching may rely on more effortful executive processes. Methods for measuring clustering and switching (Troyer et al., 1997) have been proposed and showed with correlational analyses that both cluster size and switching variables highly contribute to performance in semantic fluency, while phonemic fluency is more switching-dependant (see also Robert et al., 1998).

On the basis of Troyer's scoring system -cluster size and number of switches-, numerous researches have been conducted to evidence potential dissociations between switching and clustering abilities in a large variety of pathological conditions. Focal frontal lesions for instance seem to impair switching in phonemic fluency while focal temporal lesions would impair clustering in semantic fluency (Troyer et al., 1998a). In psychiatric populations, like depressive patients, deficits in semantic fluency have been related to a reduced number of switches (Fossati et al., 2003). The clustering/switching dimensions have also been used to characterize different subcortical syndromes. In HIV-associated dementia, switching in phonemic verbal fluency would be selectively affected (Woods et al., 2004). Dementia with Parkinson's disease impairs both phonemic and semantic switches but not semantic cluster size (Troyer et al., 1998b). In Huntington's disease, phonemic switching deficits increasing all along the course of the disease have been reported with a cluster size remaining stable (Rich et al., 1999; Ho et al., 2002). However, other authors found that patients with multiple sclerosis presented higher cluster size scores than patients with 
Huntington's disease and Parkinson's disease dementia (Tröster et al., 1998).

Some studies have also been conducted to determine whether differences in clustering and switching parameters could be evidenced between $\mathrm{AD}$ and other types of dementia. Discrepant results have been reported. Troyer et al. (1998b) found that semantic cluster size was reduced in AD but not in Parkinson dementia while Tröster et al. (1998) showed that cluster size and switches were reduced in both $\mathrm{AD}$ and Parkinson dementia. On the other hand, Epker et al. (1999) reported no differences in semantic cluster size between elderly controls, AD patients, Parkinson's disease patients with or without dementia. Previous studies comparing $\mathrm{AD}$ patients and normal elderly on the qualitative variables of animal category fluency have led to different patterns of results. March and Pattison (2006) reported deficits limited to cluster size while Epker et al. (1999) reported a lower number of switches. However, the majority of studies (Beatty et al., 1997; Troyer et al., 1998b; Tröster et al., 1998) found that both cluster size and switches were reduced in AD patients. Methodological and sampling differences across these studies may be highlighted and could partly explain the discrepancy of results.

The results in favour of an earlier switching impairment in the course of AD would be consistent with the results reported by Perry et al. (2000). These authors examined the occurrence of deficits in attention and other cognitive domains such as episodic memory, semantic memory and visuo-spatial function in the course of AD. The results showed that problems with response inhibition and attentional switching occur in the very early stages of the disease and that they were more prevalent than semantic memory deficits. The authors concluded that attention deficits may occur at earlier stage than semantic memory deficits and that the two are partially independent. In addition, a recent study on healthy adults presenting a genetic risk factor for AD by carrying the apolipoprotein E $\varepsilon 4$ allele (APOE $\varepsilon 4$ ) has been conducted by Rosen et al. (2005). Using a ten-minute category fluency task, this study 
reported, not only a reduced verbal production in APOEE4 carriers compared to the non APOEE4 carriers, but also longer between-cluster retrieval times, i.e. a larger time to access clusters. Moreover, the between-cluster retrieval time was significantly correlated to the Trail Making Test (Form B-Form A), a measure classically considered as an index of switching ability. The authors concluded that reduced attentional capacity in the APOE\&4 carriers may have raised difficulties in shifting attention among subcategories during the semantic fluency task.

The purpose of the present study was to explain the particularly early reduction observed in semantic verbal fluency in the prodromal phase of AD by considering switching and clustering processes. With the longitudinal follow-up of the Paquid study, it has been possible to measure switching and clustering processes in the animal category of the IST in future AD subjects who would develop dementia 5 years later, 2 years later as well as at the clinical onset of dementia syndrome. We hypothesised that switching processes deteriorate earlier than clustering processes in the course of the disease. The deterioration of switching processes would explain the early decline in semantic verbal fluency observed in the predementia stages of the disease while both switching and clustering deficits would be present when reaching the stage of dementia.

\section{Methods}

\section{Paquid study.}

The detailed methodology of the Paquid study has been presented previously (Dartigues et al., 1992). Briefly, Paquid is a longitudinal population-based study conducted on 3777 elderly adults living at home in the South-western part of France. Subjects were interviewed at baseline (T0), then every two or three years (T3, T5, T8, T10, T13, T15, T17), by means of 
Verbal fluency in preclinical AD

demographic questionnaires, psychometric tests and health scales, administered at home by a psychologist. After suspicion of dementia by the psychologist, a neurologist confirmed the dementia syndrome and established an etiological diagnosis using DSMIII-R criteria (American Psychiatric Association, 1987). The diagnosis of possible or probable AD was made according to NINCDS-ADRDA (National Institute for Communicative Disorders and Stroke-Alzheimer's Disease and related Disorders association) criteria (McKhann et al., 1984). All participants have given informed consent to the study, which was approved by the local Ethics Committee.

\section{Subjects.}

Patients: Incident cases of possible and probable AD diagnosed at T10 or T15 were selected. To be included in the study sample, the patients had to have completed the animal category of the IST at the visit of dementia diagnosis (called hereinafter visit D) and at the two preceding visits ( 2 years before diagnosis called visit D-2 and 5 years before diagnosis called visit D-5) which is T5, T8 for incident cases diagnosed at T10 and T10, T13 for incident cases diagnosed at T15. Among the 84 incident cases diagnosed at T10, 36 patients had completed the IST at T5, T8 and T10. For the 53 incident cases diagnosed at T15, only 15 had completed the task at T10, T13 and T15.

Controls: Control elderly subjects were selected among the subjects of the Paquid cohort seen at the same follow-up visit as the patients. Each $\mathrm{AD}$ case was closely matched for age $( \pm 5$ years), sex and education (3 classes: no diploma, primary degree, secondary degree and plus) to 2 non-demented control subjects. The elderly controls matched with the incident AD cases diagnosed at T10 had to be free of dementia at T10 and had to have completed the animal verbal fluency at T5, T8 and T10. The elderly controls matched with the incident AD cases diagnosed at T15 had to be free of dementia at T15 and had to have completed the animal 
verbal fluency at T10, T13 and T15. Non-demented subjects with Parkinson disease or depressive symptomatology documented by a score $\geq 17$ for men and $\geq 23$ for women on the Center for Epidemiologic Studies Depression Scale (Radloff, 1977) were excluded. In addition, to be included control subjects had also to be free of dementia or Parkinson disease at the next follow-up (i.e. T13 or T17) to avoid the inclusion of subjects in the a prodromal phase of dementia. Following this procedure, 72 control subjects were selected among the 561 normal subjects eligible at T10 and 30 control subjects were selected among the 412 normal subjects eligible at T15. The analyses were conducted on a total sample of 153 subjects: 51 incident cases of $\mathrm{AD}$ and 102 non-demented control subjects matched for age, sex, and educational level.

\section{Qualitative analysis of the animal verbal fluency.}

The IST (Isaacs and Kennie, 1973) is a semantic verbal fluency test requiring the subject to produce as many words as possible belonging to a semantic category in one minute. Four categories are successively used: colours, animals, fruits and cities. In the present study, we selected the animal category to estimate clustering and switching parameters according to the qualitative analysis procedure proposed by Troyer et al. (1997). Clusters consisted of successively generated words belonging to a same subcategory as specified below. Clustering was assessed according to the 3 general criteria: zoological categories, living environment and human use, based on the listing of subcategories and examples proposed by Troyer (1997). Some subcategories were added to this list due the presence of species frequently encountered in the South-western part of France (e.g. fossorial animals, noxious animals or hunted animals).

Furthermore, the two following scoring rules defined by Troyer were strictly applied: (i) in the case where two categories overlapped, with some items belonging to both categories, 
some items belonging exclusively to the first category and some items belonging exclusively to the second category, the overlapping items were assigned to both categories; (ii) in the case where smaller clusters were embedded within larger ones or two categories overlapped, but all items could correctly be assigned to a single category, only the larger, common category was considered. Considering the cluster size, two related words constitute a cluster size of 1 , three related words constitute a cluster size of 2 and so forth. A single word constitutes a cluster size of 0 . The repetitions were excluded for the calculation of cluster size.

For each assessment, 3 scores were calculated including the total number of correct words generated, the mean cluster size and the number of switches. The total number of words corresponded to the sum of all correct words produced, excluding intrusions and repetitions. A word corresponding to a superordinate subcategory (e.g. bird) was excluded from the total number of correct words if exemplars belonging to this subcategory were given. The mean cluster size for a subjects' production was calculated as follows: sum of clusters size divided by the number of clusters. The number of switches was calculated as the total number of transitions between clusters, including single words.

The scoring rules defined above slightly differed from those of Troyer considering intrusions and repetitions, which were not included in the scores for the present study. The ability to explore different new semantic fields was focused in this analysis.

\section{Statistical analyses.}

The inter-rater reliability was assessed by comparing the scoring performed by two independent raters who were blind of information concerning the clinical status of the subjects. The degree of concordance between the two raters for the variables is estimated by the R intraclass correlation coefficient. This coefficient was calculated separately for the mean cluster size and the raw number of switches, on the whole sample and pooling the data of the 
3 visits (D-5, D-2 and D). For the mean cluster size and the number of switches, R coefficients were respectively 0.9360 and 0.9944 . The concordance is estimated as very good if $\mathrm{R}>.91$ and good if .91 > R > .71 (Fermanian, 1984).

Cases and controls were first compared on demographic variables using Student ttests. We computed means and standard deviations of animal verbal fluency performances and the differences between future $\mathrm{AD}$ subjects and control subjects were tested by a logistic regression model adjusted for age, gender and education. Furthermore we used Hedges' $\hat{g}$ to measure the strength of the relationship between cases and controls. To study the evolution of each indicator which are quantitative variables, a linear regression model was performed. As several measures were collected across time for each subject, the measures are not independent. To deal with this intrasubject correlation, a random effects linear model was used. In this analysis, the parameter of interest is the slope of the measure evolution. This intrasubject correlation was modeled by two independent subject specific random effects: a random intercept and a random slope. Statistical analyses were performed with SAS statistical package release 8.2 (SAS Institute Inc., Cary, NC, USA).

\section{Results}

\section{Characteristics of the samples studied.}

The characteristics of the sample are presented in table 1 . The mean age of the subjects at the baseline (T0) of the study was respectively 75.42 years $(\mathrm{SD}=5.36)$ for the case group and 73.90 years $(\mathrm{SD}=4.34)$ for the control group. The future $\mathrm{AD}$ patients group and the control group were comparable, for gender and education level as shown in table 1 . The AD cases tended to be older than controls even if the difference was not statistically significant $(\mathrm{p}=$ 0.06). The MMSE score (Folstein et al., 1975) of the future AD subjects was significantly 
Verbal fluency in preclinical AD

lower 5 years before dementia incidence than that of controls.

Insert table 1 about here

\section{Animal verbal fluency performances at $D-5, D-2$ and $D$.}

The quantitative and qualitative parameters of the animal verbal fluency are shown in table 2 . First of all, the logistic regression model indicated that the total number of words generated was already lower in the future AD patients than in controls 5 years before dementia onset. Neither repetitions, nor intrusion rates differed between the two samples.

Regarding the mean cluster size scores, the logistic regression analysis revealed no significant differences between the two groups at any time of the follow-up. In contrast, the future $\mathrm{AD}$ patients presented lower switching scores than elderly controls at all the visits: 5 and 2 years before dementia onset and at dementia onset.

Insert table 2 about here

Evolution of the qualitative measures in the future AD subjects.

In the future $\mathrm{AD}$ patients group, the evolution of the mean number of words, the mean cluster size and the mean number of switches were analyzed using a random effects linear regression model (figure 1). The slope of the total number of words significantly decreased over time $(\mathrm{p}<0.001)$. The evolution of the cluster size over time was not significant $(\mathrm{p}=0.09)$ but the switching score showed a significant decrease between D-5 and D ( $\mathrm{p}=0.002)$.

Insert figure 1 about here 
Semantic subcategories explored by the future AD subjects at $D-5$ and $D$.

Finally, we qualitatively examined the semantic fields explored by the future AD subjects in the preclinical and clinical phases. Figure 2 describes the rate of occurrence of each subcategory at the D-5 visit and the dementia incidence visit. As may be seen, for the "living environment field" criterion, the most frequently generated categories were farm animals and Africa animals. For the "zoological" criterion, the subcategory of birds was the most frequent while for the "human use" criterion pets and hunted animals were the most frequent. All the semantic subcategories explored at the D-5 have also been explored at the D visit but to a lesser degree. Only 3 subcategories seemed to be explored at the dementia incidence as frequently as at D-5: farm animals, pets and hunted animals.

Insert figure 2 about here

\section{Discussion}

Our results first confirm the impairment of category fluency task performance in the prodromal phase of AD. A previous analysis from the Paquid study (Auriacombe et al., 2006) showed that repetitions and intrusions rates were not different between future AD and control subjects and thus could not explain the early deficit on the animal verbal fluency. The present qualitative analysis of the verbal fluency production provides a possible explanation for this decline. This study is the first to measure clustering and switching parameters of verbal fluency in the preclinical phase of AD with a longitudinal design. Our analysis evidenced significant qualitative differences between the future $\mathrm{AD}$ patients and the control subjects, including 5 years before the dementia onset. Two main results came out of this analysis. Firstly, we have shown a switching score lower in the future AD subjects than in controls 5 
years before dementia onset. The effect size index calculated to estimate the importance of the difference evidenced between the groups corroborates this result. Secondly, a significant decline in this parameter all along the prodromal phase until the clinical dementia diagnosis was observed. Indeed, the random effects linear regression model showed a significant decrease over time for the switching measure only. In addition, the evolution of the effect size also supports this conclusion: the effect size indices related to the differences in the number of switches increase notably from the D-5 visit until dementia onset whereas the effect size indices for the cluster size remain very weak across the successive time points. Therefore, the most likely explanation for the decline in category verbal fluency performance observed in the predementia phase would be an executive deficit rather than a semantic deficit. This switching deficit increases in a progressive manner until the dementia diagnosis. In contrast, the results suggest that once a specific subcategory has been activated, the future AD patients seem to be able to generate exemplars belonging to this subcategory as efficiently as do the control subjects. Moreover, the descriptive analysis of the semantic subcategories generated by the AD subjects suggests a possible reduction of the semantic fields explored in the dementia phase compared with the predementia phase, with only a limited number of subcategories being still activated. The progressive increase in switching deficits all along the course of the disease could explain this qualitative change in the semantic fields explored. The subcategories the least frequently activated by environmental conditions seem to be less explored by the AD subjects, while subcategories like "pets," "farm animals" or "hunted animals", more naturally reinforced by environment are still well accessed by the patients.

Such dissociation between clustering and switching measures has already been shown in patients with Huntington's disease followed-up during a 5-year period who exhibited an isolated switching deficit in phonemic fluency (Rich et al., 1999; Ho et al., 2002). In AD, the results previously reported have been somewhat inconsistent. Some studies concluded for the 


\section{Verbal fluency in preclinical AD}

existence of both clustering and switching deficits (Beatty et al., 1997; Troyer et al., 1998b; Tröster et al., 1998). Nonetheless, a single component deficit has also been reported, with a study evidencing a switching deficit (Epker et al., 1999) and another study showing a clustering deficit (March and Pattison, 2006) in animal fluency but which was not replicated in the supermarket fluency task. The difference in dementia severity of the patients included in these studies has been proposed to explain results discrepancy (March and Pattison, 2006). In our study, the subjects diagnosed with dementia at the D-visit were in the mild phase of dementia (mean MMSE: 20.8) and thus were less severe patients than those included in Beatty's study (Beatty et al., 1997) who presented a double deficit. Troyer and Moscovitch (2006) suggested that the dissociation between the 2 components, clustering and switching, could occur only during the early stages of $\mathrm{AD}$, when neuropathological changes in the brain are limited. However, according to these authors, the clustering process would be the first altered in the course of $\mathrm{AD}$ and secondarily, switching process would be affected in more advanced phases of the disease. Demographic variables and sampling differences could also partly explain the discrepant results of the literature. The present study has the advantage to show results on a large sample (153 subjects) contrarily to previous studies which have been conducted in smaller samples $(\mathrm{n}<80)$. Concerning age and education, due to its populationbased design, older and lower educated subjects are more represented in our study. Nevertheless, the total word production at the dementia incidence found in the present study seems to be in the range of that reported in the other studies.

In order to approach the predementia period, Murphy et al. (2006) compared verbal fluency patterns in AD patients, amnestic mild cognitive impairment (aMCI) subjects and normal controls. Regarding the clustering measure, the results showed that aMCI subjects exhibited intermediary performances between $\mathrm{AD}$ and control subjects while the switching measure was not different between the aMCI group and the control group. Thus, the authors 
concluded for a degradation of semantic networks in the preclinical phase of AD. It is important to highlight a marked difference between this research and the current work. Murphy et al.'s study (2006) is based on the results obtained on a small sample of MCI subjects $(\mathrm{n}=33)$ among which 19 were selected from newspaper advertisements. Although these subjects were described as presenting memory complaints and slight deficits in an episodic memory task, the evolution towards dementia of such subjects is quite uncertain and one can wonder whether these subjects can be really considered as predemented subjects. The longitudinal design of this study allowed to capture genuine premorbid patients and not only subjects presenting a potential risk of developing dementia in the subsequent years. This important methodological difference in the sampling procedure leads us to contrasting results which support the hypothesis of executive processes impairment in the early course of AD, with relatively preserved semantic processes. In this way, the present work provides new elements in the study of the predementia phase of AD.

Nonetheless, some advantages but also limitations can be highlighted regarding Troyer's two-component model used in the present study and its scoring system. The good interrater reliability is one of the psychometric properties of the model ( $r=.93$ and .99 in our study). The scoring rules provided are clear and relatively easy to apply. Even so, large semantic categories and subcategories defined by Troyer failed to capture all the word associations which occurred in the animal fluency output. Most of the authors who have used this method have added some amendments regarding the clustering scoring. For example, Gierski and Ergis (2004) added a modification to the subcategories listing: the "associated words in the French language" criterion (e.g. crow/fox, associated in the Fables of La Fontaine). Abwender et al. (2001) added a phonemic clustering criterion in semantic fluency and a semantic clustering criterion in phonemic fluency. Furthermore, he distinguishes taskconsistent clustering (e.g. semantic clustering on semantic fluency) and task-discrepant 
clustering (e.g. phonemic clustering on semantic fluency). He argues that task-discrepant clustering, even if less frequent, reflects a more deliberate strategy utilization than taskconsistent clustering, particularly on semantic fluency, where passive automatic wide spread activation underlies word access.

Concerning the scoring measures, some limitations can be observed from our data in particular with the parameter related to "cluster size". Indeed, data distribution shows important variability of this measure due to the occurrence for some subjects of patterns of performance consisting in large single clusters with no switch. This type of variability could have reduced statistical effects and consequently, could have masked a real clustering deficit. However this variability was found in the two groups of subjects.

Other alternative scoring methods have been proposed. To avoid the problem due to the relationship between the absolute number of switches and the total number of generated words, Tröster et al. (1998) proposed to use ratios, i.e. number of switches adjusted on the total word production. However both Tröster et al. (1998) and Troyer and Moscovitch (2006) concluded that this scoring method produced no meaningful information. Abwender et al. (2001) suggested a modified scoring system by distinguishing two types of switching: the "real switches" (switches between multi-word clusters) and the "hard switches" (switches between isolated words). According to these authors only "real switches" underlie strategic executive processes while "hard switches" would rather be a measure of general processing speed. For Mayr (2002), the switching measure is somewhat ambiguous. A general decline in processing speed would entail a slower retrieval of words either within or between clusters resulting in a reduced switching score, which cannot be distinguished from a specific switching deficit. Thus he suggested to consider time measures and to compare the retrieval times for the words generated within cluster with the retrieval times between clusters. This measure was examined in APOE\&4 carrier subjects compared to non APOE\&4 carriers by 
Rosen et al. (2005) who found that only time to access clusters was longer in the APOE\&4 carriers. This between-cluster time retrieval was correlated with a switch index measure leading the authors to conclude for a switching deficit in APOE\&4 carriers.

The last but maybe the more important limitation of the two-component model proposed by Troyer has been theorised by Mayr (2002) who underlines that each act of retrieval is the result of both executive and semantic processes. Indeed, according to this author, executive processes are involved in each word retrieval, including within intra-cluster words. This interesting conception introduces the problem of measure purity of the two parameters supposed to independently measure executive and semantic processes.

However, it is generally considered that semantic processes (e.g. wide spread activation in the semantic network) are rather dominant in the clustering phase while executive processes are rather dominant in the switching phase. Relationships between switches and standard executive measures may support this conceptual view. A study on depressed patients (Fossati et al., 2003) demonstrated that the reduction in the number of switches in semantic fluency was significantly correlated with a reduced ability to shift mental set on the modified version of the Wisconsin Card Sorting Test.

Thus despite the limitations described above, the present work suggests the impairment of executive processes of verbal fluency in the preclinical phase of AD which gradually decline until the clinical diagnosis of $\mathrm{AD}$. The hypothesis of an executive impairment in the preclinical phase of AD is consistent with other studies. Reviews and metaanalyses (Bäckman et al., 2004; Bäckman et al., 2005) of both longitudinal population-based samples and studies on subjects with MCI have evidenced that together with episodic memory deficits, changes in executive functioning and perceptual speed were present in the very early phases. In the same vein, the PAQUID cohort (Amieva et al., 2005) has shown that multiple cognitive functions are affected in the premorbid phase of the AD among which executive 
Verbal fluency in preclinical AD

functions deficits. Furthermore, a recent cross-sectional study on a small group of MCI patients (Gualtieri and Johnson, 2005) demonstrated that tests of memory, processing speed and cognitive flexibility were the most important discriminators between normal controls and MCI patients.

In summary, this study has confirmed the early decline in semantic verbal fluency performances in premorbid subjects, 5 years before the clinical diagnosis of AD. The absence of differences in repetitions and intrusions rates has confirmed that inhibitory or up-dating processes regulating working-memory do not significantly contribute to this early decline. However, the qualitative analysis based on clustering and switching measures of verbal fluency supports the hypothesis of an early and progressive decline in switching processes. This impairment could explain the diminished semantic fluency performances of future AD patients long before the clinical diagnosis of $\mathrm{AD}$. Switching processes in the verbal fluency paradigm may involve the ability to process a strategic search, to disengage from a recently activated category and to activate a new one. Further researches are needed to discriminate which of these switching components is the critical one. 
Verbal fluency in preclinical AD

\section{Acknowledgements}

The PAQUID project was funded by: ARMA (Bordeaux); Caisse Nationale d'Assurance Maladie des Travailleurs Salariés (CNAMTS); Conseil Général de la Dordogne; Conseil Général de la Gironde; Conseil Régional d'Aquitaine; Fondation de France; France Alzheimer (Paris); GIS Longévité; Institut National de la Santé et de la Recherche Médicale (INSERM); Mutuelle Générale de l'Education Nationale (MGEN); Mutualité Sociale Agricole (MSA); NOVARTIS Pharma (France); SCOR Insurance (France). 


\section{References}

ABWENDER DA, SWAN JG, BOWERMAN JT and CONNOLLY SW. Qualitative analysis of verbal fluency output: review and comparison of several scoring methods. Assessment, 8: 323-338, 2001.

AMERICAN PSYCHIATRIC ASSOCIATION. DSM-III-R: Diagnostic and Statistical Manual of Mental Disorders DSM-III-R (Text Revision) (4th ed, ). Washington, D.C.: In American Psychiatric Association (Ed), 1987.

AMIEVA H, JACQMIN-GADDA H, ORGOGOZO JM, LE CARRET N, HELMER C, LETENNEUR L, BARBERGER-GATEAU P, FABRIGOULE $C$ and DARTIGUES JF. The 9 year cognitive decline before dementia of the Alzheimer type: a prospective population-based study. Brain, 128: 1093-1101, 2005.

AURIACOMBE S, LECHEVALLIER N, AMIEVA H, HARSTON S, RAOUX N and DARTIGUES JF. A longitudinal study of quantitative and qualitative features of category verbal fluency in incident Alzheimer's disease subjects: results from the PAQUID study. Dementia and Geriatric Cognitive Disorders, 21: 260-266, 2006.

BÄCKMAN L, JONES S, BERGER AK, LAUKKA EJ and SMALL BJ. Multiple cognitive deficits during the transition to Alzheimer's disease. Journal of Internal Medecine, 256: 195-204, 2004.

BÄCKMAN L, JONES S, BERGER AK, LAUKKA EJ and SMALL BJ. Cognitive impairment in preclinical Alzheimer's disease: a meta-analysis. Neuropsychology, 19: 520-531, 2005.

BEATTY WW, TESTA JA, ENGLISH S and WINN P. Influences of clustering and switching on the verbal fluency performance of patients with Alzheimer's Disease. Aging, Neuropsychology and Cognition, 4: 273-279, 1997.

DARTIGUES JF, GAGNON M, BARBERGER-GATEAU P, LETENNEUR L, COMMENGES D, SAUVEL C, MICHEL $P$ and SALAMON R. The Paquid epidemiological program on brain ageing. Neuroepidemiology, 11: 14-18, 1992.

EPKER MO, LACRITZ LH and CULLUM CM. Comparative analysis of qualitative verbal fluency performance in normal elderly and demented populations. Journal of Clinical and Experimental Neuropsychology, 21: 425-434, 1999.

FERMANIAN J. Mesure de l'accord entre deux juges: cas quantitatif. Revue 
d'Epidémiologie et de Santé Publique, 32: 408-413, 1984.

FOLSTEIN MF, FOLSTEIN SE and MCHUGH PR. "Mini-mental state". A practical method for grading the cognitive state of patients for the clinician. Journal of Psychiatry Research, 12: 189-198, 1975.

FOSSATI P, LE BASTARD G, ERGIS AM and ALLILAIRE JF. Qualitative analysis of verbal fluency in depression. Psychiatry Research, 117: 17-24, 2003.

GIERSKI F and ERGIS AM. Les fluences verbales : aspects théoriques et nouvelles approches. L'Année Psychologique, 104: 331-360, 2004.

GUALTIERI CT and JOHNSON LG. Neurocognitive testing supports a broader concept of mild cognitive impairment. American Journal of Alzheimer's Disease and Other Dementias, 20(6), 359-366, 2005.

HENRY JD, CRAWFORD JR and PHILLIPS LH. Verbal fluency performance in dementia of the Alzheimer's type: a meta-analysis. Neuropsychologia, 42: 1212-1222, 2004.

HO AK, SAHAKIAN BJ, ROBBINS TW, BARKER RA, ROSSER AE and HODGES JR. Verbal fluency in Huntington's disease: a longitudinal analysis of phonemic and semantic clustering and switching. Neuropsychologia, 40: 1277-1284, 2002.

ISAACS B and KENNIE AT. The Set test as an aid to the detection of dementia in old people. British Journal of Psychiatry, 123: 467-470, 1973.

MARCH EG and PATTISON P. Semantic verbal fluency in Alzheimer's disease: approaches beyond the traditional scoring system. Journal of Clinical and Experimental Neuropsychology, 28: 549-566, 2006.

MAYR U. On the dissociation between clustering and switching in verbal fluency: comment on Troyer, Moscovitch, Winocur, Alexander and Stuss. Neuropsychologia, 40: 562-566, 2002.

MCKHANN G, DRACHMAN D, FOLSTEIN M, KATZMAN R, PRICE D and STADLAN EM. Clinical diagnosis of Alzheimer's disease: report of the NINCDSADRDA Work Group under the auspices of Department of Health and Human Services Task Force on Alzheimer's Disease. Neurology, 34: 939-944, 1984.

MURPHY KJ, RICH JB and TROYER AK. Verbal fluency patterns in amnestic mild cognitive impairment are characteristics of Alzheimer's type dementia. Journal of the 
International Neuropsychological Society, 12: 570-574, 2006.

PERRY RJ, WATSON $\mathrm{P}$ and HODGES JR. The nature and staging of attention dysfunction in early (minimal and mild) Alzheimer's disease: relationship to episodic and semantic memory impairment. Neuropsychologia, 38: 252-271, 2000.

RADLOFF LS. The CES-D scale: A self-report depression scale for research in the general population. Applied Psychological Measurement, 1: 385-401, 1977.

RASKIN SA, SLIWINSKI M and BOROD JC. Clustering strategies on tasks of verbal fluency in Parkinson's disease. Neuropsychologia, 30: 95-99, 1992.

RICH JB, TROYER AK, BYLSMA FW and BRANDT J. Longitudinal analysis of phonemic clustering and switching during word-list generation in Huntington's disease. Neuropsychology, 13: 525-531, 1999.

ROBERT PH, LAFONT V, MEDECIN I, BERTHET L, THAUBY S, BAUDU C and DARCOURT G. Clustering and switching strategies in verbal fluency tasks: comparison between schizophrenics and healthy adults. Journal of the International Neuropsychological Society, 4: 539-546, 1998.

ROBERT PH, THAUBY S, MIGNECO V, CHAIX I, CACI H, BENOIT M, PRINGUEY D and DARCOURT G. Différenciation de sous-types de troubles schizophréniques en fonction des performances au test de fluence verbale. Encephale, 22: 435-442, 1996.

ROSEN MV, SUNDERLAND T, LEVY J, HARWELL A, MCGEE L, HAMMOND C, BHUPALI D, PUTNAM K, BERGESON J and LEFKOWITZ C. Apolipoprotein E and category fluency: evidence for reduced semantic access in healthy normal controls at risk for developing Alzheimer's disease. Neuropsychologia, 43: 647-658, 2005.

TRÖSTER AI, FIELDS JA, TESTA JA, PAUL RH, BLANCO CR, HAMES KA, SALMON DP and BEATTY WW. Cortical and subcortical influences on clustering and switching in the performance of verbal fluency tasks. Neuropsychologia, 36: 295304, 1998.

TROYER AK, MOSCOVITCH M and WINOCUR G. Clustering and switching as two components of verbal fluency: evidence from younger and older healthy adults. Neuropsychology, 11: 138-146, 1997.

TROYER AK, MOSCOVITCH M, WINOCUR G, ALEXANDER MP and STUSS D. Clustering and switching on verbal fluency: the effects of focal frontal- and temporallobe lesions. Neuropsychologia, 36: 499-504, 1998a. 
TROYER AK, MOSCOVITCH M, WINOCUR G, LEACH L and FREEDMAN M. Clustering and switching on verbal fluency tests in Alzheimer's and Parkinson's disease. Journal of the International Neuropsychological Society, 4: 137-143, 1998b.

TROYER AK and MOSCOVITCH M. Cognitive processes of verbal fluency tasks. In Taylor \& Francis (Ed), The Quantified process approach to neuropsychological assessment New York, United States, 2006, ch.10.

WOODS SP, CONOVER E, RIPPETH, JD, CAREY CL, GONZALEZ R, MARCOTTE TD, HEATON RK and GRANT I. Qualitative aspects of verbal fluency in HIVassociated dementia: a deficit in rule-guided lexical-semantic search processes? Neuropsychologia, 42: 801-809, 2004. 
Verbal fluency in preclinical AD

Table 1. Demographic characteristics and mental status for the future AD subjects and normal controls.

\begin{tabular}{|c|c|c|c|c|}
\hline & $\begin{array}{c}\text { Future AD } \\
\text { subjects } \\
(n=51)\end{array}$ & $\begin{array}{l}\text { Control } \\
\text { subjects } \\
(\mathrm{n}=102)\end{array}$ & $\mathrm{P}$ value $*$ & Size effect $* *$ \\
\hline Age at D-5 (mean (SD)) & $81.98(4.90)$ & 80.49 (3.59) & 0.06 & 0.36 \\
\hline \multicolumn{5}{|l|}{ Education (n (\%)) } \\
\hline No diploma & $22(43.1 \%)$ & $44(43.1 \%)$ & & \\
\hline Primary degree & $19(37.3 \%)$ & $38(37.3 \%)$ & & \\
\hline Secondary degree and plus & $10(19.6 \%)$ & $20(19.6 \%)$ & & \\
\hline \multicolumn{5}{|l|}{$\operatorname{Sex}(n(\%))$} \\
\hline male & $20(39.2 \%)$ & $40(39.2 \%)$ & & \\
\hline female & $31(60.8 \%)$ & $62(60.8 \%)$ & & \\
\hline MMSE at D- ${ }^{a}$ visit (mean (SD)) & $25.57(3.10)$ & $27.58(2.02)$ & $<0.0001$ & 0.82 \\
\hline MMSE at D- ${ }^{b}$ visit (mean (SD)) & $24.78(2.77)$ & $27.13(2.21)$ & $<0.0001$ & 0.97 \\
\hline MMSE at $\mathrm{D}^{\mathrm{c}}$ visit (mean (SD)) & $20.8(3.86)$ & $26.66(2.19)$ & $<0.0001$ & 2.04 \\
\hline
\end{tabular}

MMSE $=$ Mini-Mental State Examination

* Student t-test

** Hedges' $\hat{g}$ effect size index

${ }^{\mathrm{a}} \mathrm{D}-5,5$ years before dementia onset

${ }^{b} \mathrm{D}-2,2$ years before dementia onset

${ }^{\mathrm{c}} \mathrm{D}$, time of dementia onset 
Verbal fluency in preclinical AD

Table 2. Animal verbal fluency performances and qualitative variables (mean and SD) among the future AD subjects and the control subjects at the D-5, D-2 and D visits.

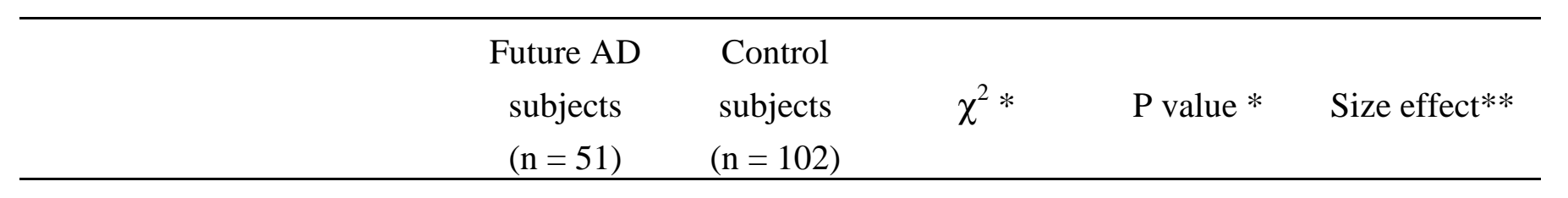

Total number of words

$\begin{array}{lrrrrr}\text { D-5 } & 13.08(3.82) & 15.87(4.67) & 12.31 & 0.0005 & 0.63 \\ \text { D-2 } & 11.75(3.19) & 15.63(4.36) & 23.67 & <0.0001 & 0.96 \\ \text { D } & 8.98(3.31) & 15.53(5.04) & 32.5 & <0.0001 & 1.44\end{array}$

Repetition rates

$\begin{array}{llllll}\text { D-5 } & 0.61(1.30) & 0.71(1.29) & 0.02 & 0.8744 & 0.08 \\ \text { D-2 } & 0.37(0.75) & 0.66(1.12) & 1.79 & 0.1807 & 0.28 \\ \text { D } & 0.37(0.92) & 0.58(1.26) & 0.61 & 0.4329 & 0.18\end{array}$

Intrusions rates

$\begin{array}{lllccc}\text { D-5 } & 0 & 0 & - & & \\ \text { D-2 } & 0.04(0.28) & 0.20(0.14) & 0.42 & 0.5169 & 0.81 \\ \text { D } & 0.22(0.97) & 0.01(0.10) & 2.21 & 0.1371 & 0.37\end{array}$

Mean cluster size

$\begin{array}{llllll}\text { D-5 } & 1.94(1.25) & 2.16(1.63) & 0.79 & 0.3745 & 0.14 \\ \text { D-2 } & 1.83(1.62) & 1.95(1.08) & 0.52 & 0.4713 & 0.09 \\ \text { D } & 1.67(1.66) & 1.73(1.13) & 0.02 & 0.8738 & 0.04\end{array}$

Number of switches

$\begin{array}{lllcrr}\text { D-5 } & 4.16(2.04) & 4.99(2.53) & 3.97 & 0.0465 & 0.35 \\ \text { D-2 } & 4.04(2.10) & 4.92(2.08) & 5.01 & 0.0252 & 0.42 \\ \text { D } & 2.96(1.80) & 5.42(2.55) & 27.35 & <0.0001 & 1.05\end{array}$

*Logistic regression model with Wald $\chi^{2}$

** Hedges' $\hat{g}$ effect size index 
Verbal fluency in preclinical AD

Figure 1. Evolution of the total number of words, the mean cluster size and the number of switches between the D-5 and D visits among the future AD subjects (random effects linear regression model).

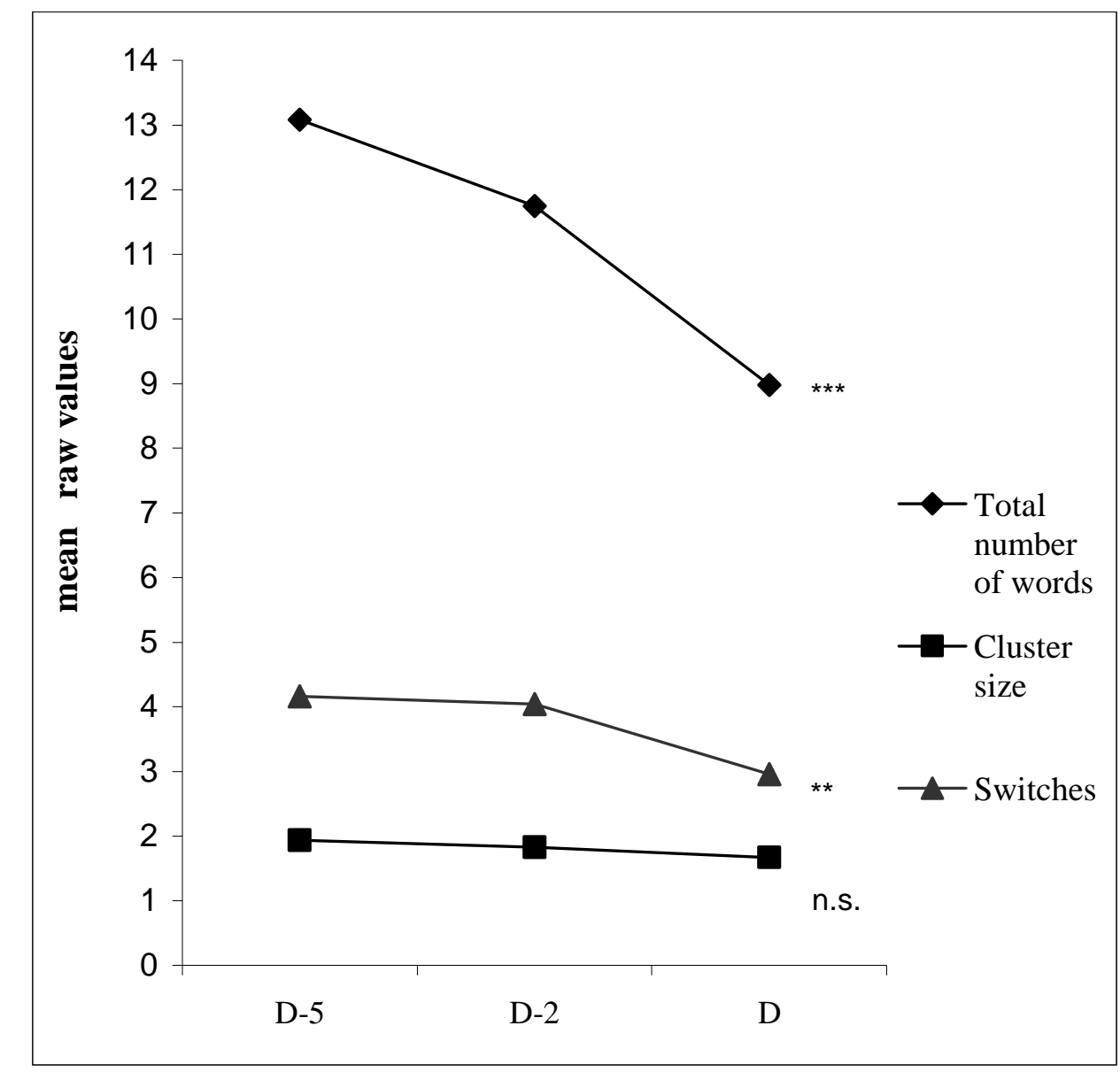

$* * * \mathrm{p}<0.0001 ; * * \mathrm{p}=0.0016$ 
Verbal fluency in preclinical AD

Figure 2. Percentage of AD subjects $(n=51)$ having explored the different subcategories at the D-5 and D visits.

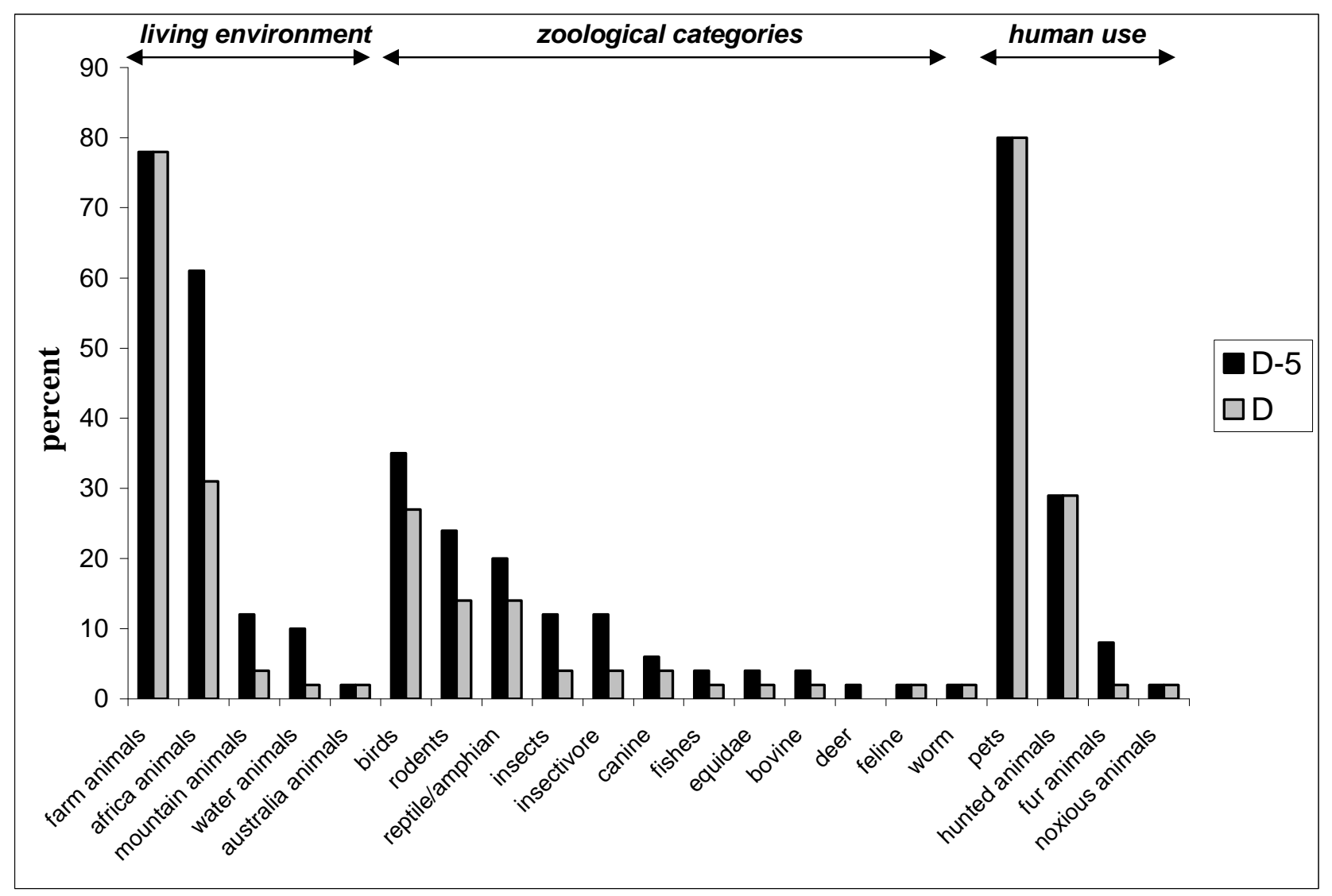

